\title{
Beyond "Cold" Cognition: Exploring Cognitive Control of Emotion as a Risk Factor for Psychosis
}

\author{
Laura M. Tully • Tara A. Niendam
}

Published online: 19 June 2014

(C) Springer International Publishing AG 2014

\begin{abstract}
The past 20 years of research examining psychosis risk factors has predominantly focused on "cold" cognitive (i.e., non-affective) processes. Despite identification of potential cognitive and associated brain-based vulnerability markers, our ability to identify those individuals at highest risk for future psychosis has not substantially improved. Consequently, researchers have begun to examine emotionprocessing deficits as potential psychosis vulnerability markers. Here we propose that a particular emotionprocessing domain, cognitive control of emotion, is a potential transdiagnostic mechanism underlying the heterogeneity of clinical presentation and psychosocial impairments observed in high-risk populations. Recent investigations indicate impaired cognitive control of emotion is observable across the psychosis spectrum and relates to important clinical and psychosocial outcomes relevant to the high-risk state. Moreover, preliminary evidence indicates treatment interventions aimed at improving cognitive control of emotion could reduce psychotic symptoms and improve functioning. We highlight gaps in current knowledge and propose five key avenues for future investigations.
\end{abstract}

Keywords Psychosis risk $\cdot$ Prodromal $\cdot$ Psychosis continuum $\cdot$ Cognitive control $\cdot$ Emotion regulation . Emotional interference $\cdot$ Social functioning

L. M. Tully $\cdot$ T. A. Niendam

Psychiatry and Behavioral Sciences, University of California, Davis, Sacramento, CA 95817, USA

L. M. Tully

e-mail:1mtully@ucdavis.edu

L. M. Tully $\cdot$ T. A. Niendam $(\bowtie)$

UC Davis Imaging Research Center,

4701 X Street, Suite E, Sacramento, CA 95817, USA

e-mail: tniendam@ucdavis.edu

\section{Introduction}

A substantial body of research demonstrates that untreated psychotic illness is consistently associated with worse longterm patient outcomes, including more severe clinical symptoms, poorer social and role functioning, and reduced quality of life, as well as increased rates of hospitalization, suicide attempts, violence, and difficulty achieving remission once treatment is initiated $[1,2]$. In an attempt to identify individuals earlier in the development of psychotic illness, over the past 20 years researchers have explored various high-risk groups to better understand the processes that contribute to the onset of psychosis. These high-risk groups include (1) genetic high-risk (GHR) individuals who have a first-degree relative with psychosis (for reviews, see Bora et al. [3] and Shah et al. [4]); (2) "psychosis prone" (PP) individuals who score highly on psychometric measures of dimensional schizotypal personality traits [5, 6]; and (3) clinical high-risk (CHR) individuals who demonstrate a cluster of attenuated psychotic symptoms consistent with established criteria (for a review, see Wood et al. [7]). To date, high-risk research has predominantly focused on cognitive deficits and associated psychosocial impairments. Cognitive deficits across multiple domains are consistently observed in high-risk populations [8], are associated with structural and functional abnormalities in frontal and temporal regions [9], and predict symptom severity, social functioning, and transition to psychosis [8, 10-12]. These cognitive deficits are considered to reflect a domain-general impairment in cognitive control-the inhibitory and facilitatory functions necessary to maintain taskrelevant processing and goal-oriented behavior - which may represent a risk marker for future clinical and psychosocial deterioration.

However, despite the identification of candidate clinical [13, 14], cognitive [8], and brain-based [9] vulnerability markers, our ability to identify those individuals at highest 
risk for future psychotic illness has not substantially improved. Neurocognitive and clinical markers tend to have low predictive power for transition to a psychotic disorder $[3,10]$, and transition rates are relatively low-only $18-36 \%$ of CHR individuals develop a psychotic disorder within 2-3 years [7]. Moreover, CHR individuals who do not develop psychosis but continue to meet high-risk criteria have substantial ongoing clinical and functional impairment [15-19]. Indeed, multiple studies have highlighted the diagnostic heterogeneity of CHR samples $[16,20]$, particularly in the context of mood dysregulation; recent estimates indicate that more than $70 \%$ of CHR individuals have comorbid depression and/or anxiety [16].

Given the high rates of mood pathology in high-risk samples, there is growing consensus that the traditional boundary between psychotic and affective symptoms is not as clear-cut as previously thought $[21 \cdot \bullet]$ and that the current approach to risk identification, dominated by investigations of nonaffective processes and positive symptoms, may not be capturing the full breadth of domains underlying psychosis risk. A recent meta-analysis demonstrated that CHR status is strongly biased toward risk for developing non-affective psychoses (schizophrenia, schizophreniform disorder, schizoaffective disorder), compared with affective psychoses (bipolar disorder or depression with psychotic features) [22]. Failing to account for affective symptoms limits the predictive value of CHR status for developing psychosis across the spectrum of possible illness presentations, and may result in the underestimation of transition to affective psychoses within high-risk groups. Indeed, given that the intensity and severity of psychotic symptoms fluctuate over time, most likely in the context of affective symptoms and environmental stressors $[23 \bullet \cdot, 24 \bullet \cdot]$, the separation of psychotic and affective psychopathology may not be useful for predicting long-term trajectory or for developing interventions. Consistent with this, and the observed heterogeneity of current psychotic disorder categories, there are increasing calls for the conceptualization of psychosis in a dimensional model that emphasizes the interaction between the severity of psychotic and affective symptoms and their consequent effects on daily life functioning, rather than categorical transition points between psychotic and not psychotic [24••]. This is particularly relevant given the Research Domain Criteria (RDoC) initiative of the National Institutes of Mental Health (NIMH), which seeks to develop a research-driven classification system for mental disorders based on neurobiologically informed dimensions that could better parse shared and unique variance across psychiatric disorders [25]. The most radical interpretation of this would be to do away with diagnostic categories and arbitrary transition points entirely, and instead characterize an individual's psychopathology along a set of dimensions of neurobiological causal mechanisms that better account for the complex interactions of psychotic and affective symptoms so often observed in patients. However, before this radical nosological shift can even be considered, we must first delineate the common and divergent biobehavioral mechanisms of affective and psychotic symptomatology to better predict illness trajectory and identify specific treatment targets within the boundaries of the current system.

Consequently, there is renewed interest in potential transdiagnostic biobehavioral mechanisms that can account for the concurrent psychotic and mood symptoms typically observed in high-risk populations [26, 27]. One candidate domain is emotion processing. The ability to identify, understand, and use emotional information in one's environment, as well as the ability to regulate one's own emotional state, is increasingly connected to mental health and well-being in daily life and may be especially important for mood regulation and adaptive social behavior [28, 29]. Consequently, high-risk researchers have begun to examine emotion-processing deficits as potential vulnerability markers for psychosis [30].

Here we focus on a particular aspect of emotion processing that may contribute to increased risk for psychosis: cognitive control of emotion - the recruitment of cognitive control mechanisms to regulate the experience and expression of one's emotional state and to control the influence of emotional information on behavior [31]. We propose that examination of cognitive control impairments in the context of emotion regulation presents a rich — and, as yet, relatively unexploredarea for investigation of potential mechanisms underlying the heterogeneity of clinical presentation and psychosocial impairments observed during the high-risk state. This is not a comprehensive review of cognitive control of emotion as a field [see 32, 33] or the wealth of high-risk literature published to date (see Shah et al. [4] and Fusar-Poli et al. [7]). Rather, we seek to provide a timely summary of recent investigations of cognitive control of emotion deficits and associated frontallimbic neural dysfunction, specifically in the context of the psychosis risk state, in order to better direct future research and facilitate its translation to the clinical setting. Accordingly, we first present a rationale for the integration of cognition and emotion in psychosis research, briefly define and describe the neural mechanisms of cognitive control of emotion determined from studies in healthy individuals, and then review recent investigations of cognitive control of emotion and its contribution to psychological well-being and social functioning in psychotic and high-risk populations. Finally, we discuss the implications for clinical settings and suggest future research directions capable of bridging these findings to further our understanding of psychosis.

\section{Integrating Cognition and Emotion in Psychosis}

Emotion dysregulation is a hallmark of psychosis, observable in the high-risk phase preceding psychotic illness and 
predictive of symptoms and social dysfunction [13, 34, 35]. Mood and anxiety symptoms are present in the majority of CHR [16, 20] and PP individuals [36], as well as in individuals with a diagnosed psychotic disorder [37, 38]. Moreover, comorbid mood and anxiety symptoms in high-risk populations relate to interpersonal sensitivity and paranoid ideation [39], delusions [40], negative symptoms [41], increased risk for transition to psychosis [35], and poorer psychosocial functioning overall [16]. Collectively, this suggests that emotion dysregulation may be part of the psychosis vulnerability profile, possible due to reciprocal causal influence between emotion dysregulation and psychotic symptoms - that is, emotion dysregulation could give rise to psychotic symptoms, and vice versa. For example, delusional content is often representative of emotional concerns; persecutory delusions can be conceptualized as delusional threat beliefs rooted in anxiety [42], and the distressing and often traumatic nature of psychosis could give rise to emotional disorders [43]. Further, psychotic symptoms are prevalent in non-psychotic disorders, suggesting that they occur more often in the context of emotion dysregulation [21]. For example, individuals with anxiety and/or depression are more likely to report delusional thought content than individuals without mood disorders [40]. Similarly, approximately $80 \%$ of individuals who report psychotic-like experiences in the absence of a diagnosable psychotic disorder also have a diagnosis of a mood and/or anxiety disorder [44]. Psychotic and affective symptoms have also been shown to reciprocally exacerbate each other over time: in a 10-year follow-up study, persistent depressive or hypomanic symptoms were found to increase the likelihood of psychotic symptoms, and persistent psychotic symptoms were found to increase the likelihood of mood symptoms [45]. This co-expression and reciprocal relationship suggests that shared mechanisms underlie both affective and psychotic pathology $[45,46]$. Mounting evidence suggests that impaired cognitive control of emotion, mediated by dysfunctional frontal-limbic neural networks, is one such shared mechanism.

Cognitive control of emotion is fundamental for successful navigation of an inherently social world. Social interactions are affective in nature, especially interpersonal conflicts, which can be emotionally challenging and require regulation of negative emotional information for successful resolution $[47,48]$. These self-regulatory processes are reliant on cognitive control mechanisms facilitated by the cingulo-frontalparietal cognitive control network $[49,50]$. Dysfunction in the cognitive control network, particularly the lateral prefrontal cortex (LPFC), is a well-established neural impairment in schizophrenia [51•] and high-risk populations [52-54], and is thought to be a biomarker for psychotic illness [8, 51•]. However, how LPFC dysfunction confers vulnerability and manifests in the core characteristics of psychosis is unknown. The diathesis-stress model of psychosis posits that putative biomarkers such as LPFC dysfunction lead to the development and maintenance of symptoms when individuals are exposed to an environmental stressor [55]. Consistent with recent theories that social stress is one such environmental stressor for psychosis [23*0], one proposal is that LPFC dysfunction is a biological vulnerability, which, in the presence of interpersonal stressors, contributes to symptoms and social impairments via impaired cognitive control of emotion [56, 57].

\section{Cognitive Control of Emotion: Definitions and Neural Mechanisms}

Cognitive control of emotion refers to the diverse set of processes involved in the effortful control of the experience and expression of our internal emotional states, as well as the more automatic control of how external emotional information influences behavior $[31,32,58]$. Here we adopt the terminology of a recently proposed dual-process model [59•] and refer to these two key cognitive control of emotion processes as (explicit) emotion regulation (ER) and (implicit) emotion conflict adaptation (ECA), respectively (see Table 1). Investigations of ER predominantly employ reappraisal/ suppression paradigms in which ER processes are evaluated on the basis of participants' self-reported changes in their emotion experience following voluntary engagement of cognitive control of emotion mechanisms [32]. In contrast, ECA research predominantly employs paradigms that require inhibition of task-irrelevant emotional stimuli (most frequently, negatively valenced) in order to focus on task-relevant responses (e.g., the facial-emotional Stroop task [60], faceshouses task [61], emotional go/no-go task [62], and emotional flanker tasks [63]), thus ECA processes are evaluated on the basis of behavioral response patterns such as accuracy and reaction time.

Neuroimaging studies in healthy individuals demonstrate that both ER and ECA paradigms engage the same cognitive control of emotion network characterized by frontal-limbic connectivity between the domain-general cognitive control network [64] and medial prefrontal and subcortical regions associated with social and affective processing [31, 65] (see Fig. 1). The cognitive control network comprises the lateral prefrontal cortex (LPFC), particularly the dorsolateral prefrontal cortex (DLPFC), the anterior cingulate cortex (ACC), and parietal regions [66]. The DLPFC is thought to maintain rules and goals relevant to the current task, and, in the presence of conflicting information that might prompt prepotent but inappropriate responses, exert "top-down" control to direct neural processing toward the current goal $[66,67]$. The ACC appears to detect the presence of response conflict and signal the DLPFC when top-down control is needed to maintain task-relevant processing $[68,69]$, while parietal regions facilitate the shifting and orienting of attention toward 
Table 1 Explicit versus implicit cognitive control of emotion

\begin{tabular}{|c|c|c|}
\hline & Explicit ER & Implicit ECA \\
\hline Definition & $\begin{array}{l}\text { Explicit, effortful control of the experience and expression of } \\
\text { internal emotional states }\end{array}$ & $\begin{array}{l}\text { Implicit, automatic control of the influence of external } \\
\text { emotional information on decisions and behavior }\end{array}$ \\
\hline How is it assessed? & $\begin{array}{l}\text { Tasks that present emotional stimuli and instruct individuals } \\
\text { to either react naturally (reactivity trials) or to regulate their } \\
\text { emotional response to the stimuli (regulation trials) }\end{array}$ & $\begin{array}{l}\text { Tasks that require inhibition of task-irrelevant emotional } \\
\text { stimuli in order to focus on task-relevant responses. Task- } \\
\text { irrelevant emotional stimuli either presented concurrently } \\
\text { (distractors) or sequentially (primes) }\end{array}$ \\
\hline Strategies & $\begin{array}{l}\text { Adaptive: reappraisal, problem solving, acceptance } \\
\text { Maladaptive: suppression, rumination, catastrophizing, self- } \\
\text { blame }\end{array}$ & Effortful/attentional/inhibitory control \\
\hline Tasks and measures & $\begin{array}{l}\text { Reappraisal/suppression paradigm [32] } \\
\text { CERQ [75] }\end{array}$ & $\begin{array}{l}\text { Facial-emotional Stroop task [60] } \\
\text { Emotional go/no-go task [62] } \\
\text { Emotional flanker task [63] } \\
\text { Faces-houses tasks [61] }\end{array}$ \\
\hline \multicolumn{3}{|c|}{ Findings in non-psychosis psychopathology } \\
\hline Behavioral & $\begin{array}{l}\text { Maladaptive strategies associated with worry/anxiety [76], } \\
\text { depression }[77,78] \text {, and substance use [79] }\end{array}$ & $\begin{array}{l}\text { Impaired ECA associated with depression [80], anxiety [81], } \\
\text { and BPD [82] }\end{array}$ \\
\hline Neuroimaging & $\begin{array}{l}\text { Increased activation in DLPFC during ER following } \\
\text { antidepressant treatment associated with reduced } \\
\text { depressive symptoms [120] }\end{array}$ & $\begin{array}{l}\text { Reduced ACC and LPFC activation during ECA in anxiety } \\
\text { [84]; reduced DLPFC and increased amygdala activation } \\
\text { in depression [85] }\end{array}$ \\
\hline \multicolumn{3}{|c|}{ Findings in psychosis-spectrum samples } \\
\hline Behavioral & $\begin{array}{l}\text { Suppression associated with more severe paranoia [87], } \\
\text { auditory hallucinations [88], and poorer social functioning } \\
\text { [94] }\end{array}$ & $\begin{array}{l}\text { Impaired ECA associated with paranoia [99], disorganized } \\
\text { symptoms [100], and reduced positive affect [108] }\end{array}$ \\
\hline Neuroimaging & $\begin{array}{l}\text { Reduced frontal-limbic connectivity during reappraisal in } \\
\text { schizophrenia [103] and PP [104] }\end{array}$ & $\begin{array}{l}\text { Reduced LPFC, medial PFC, and ACC activation during } \\
\text { ECA in schizophrenia [105] and PP [104] }\end{array}$ \\
\hline
\end{tabular}

$A C C$ anterior cingulate cortex, $B P D$ borderline personality disorder, $C E R Q$ Cognitive Emotion Regulation Questionnaire, $D L P F C$ dorsolateral prefrontal cortex, $E C A$ emotion conflict adaptation, $E R$ emotion regulation, $L P F C$ lateral prefrontal cortex, $P F C$ prefrontal cortex, $P P$ psychosis proneness

task-appropriate stimuli [70]. During cognitive control of emotion, this network interacts with regions associated with social and affective processing, including the ventrolateral prefrontal cortex (VLPFC), ventromedial prefrontal cortex (VMPFC), insula, and amygdala, comprising a top-down regulatory frontal-limbic network in which the DLPFC and VLPFC exert inhibitory control over subcortical amygdala pathways via projections from the VMPFC, modulating emotion experience and regulating response to emotional stimuli [65].

Studies in healthy individuals suggest LPFC function is particularly relevant to an individual's response to emotional and interpersonal stressors. The less that individuals recruit LPFC regulatory mechanisms during tasks that prompt ER, the less self-regulation they report in everyday life; lower VLPFC activity during the "Cyberball" social exclusion task, in which participants play a virtual ball-tossing game with two other players who eventually exclude them from the game [71], predicts higher self-reported distress as a consequence of that exclusion $[72,73]$. This suggests that reduced engagement of LPFC mechanisms in response to social rejection reflects a failure to regulate emotions in response to interpersonal stressors that could impact social behavior. Similarly, lower VLPFC activity when viewing negative facial expressions of one's romantic partner, considered an incidental form of ER [74], predicts increased negative mood and maladaptive behavior following conflict with that partner [56]. Collectively, these data indicate that successful cognitive control of emotion is reliant on intact LPFC function and that dysfunctional engagement of lateral prefrontal control mechanisms in the presence of a social/emotional stressor could contribute to psychopathology and social deficits via impaired cognitive control of emotion $[56,57]$.

\section{Relevance of Cognitive Control of Emotion to Transdiagnostic Psychosocial Outcomes}

Consistent with this proposal, there is mounting evidence that impaired cognitive control of emotion, particularly negative emotional information, impacts a range of psychological and social outcomes and thus could be a core transdiagnostic mechanism that accounts for shared symptoms across diagnostic categories [26, 27]. Maladaptive ER strategies (e.g., suppression, rumination, self-blame, catastrophizing), commonly assessed using the Cognitive Emotion Regulation 
Fig. 1 Neural regions engaged during cognitive control of emotion. During cognitive control of emotion, the cognitive control network, comprising the dorsolateral prefrontal cortex (DLPFC), anterior cingulate cortex (ACC), and superior parietal regions, interacts with the ventrolateral prefrontal cortex (VLPFC), ventromedial prefrontal cortex (VMPFC), insula, and amygdala to form a top-down regulatory frontallimbic network in which DLPFC and VLPFC regions exert inhibitory control over subcortical amygdala pathways via projections from the VMPFC

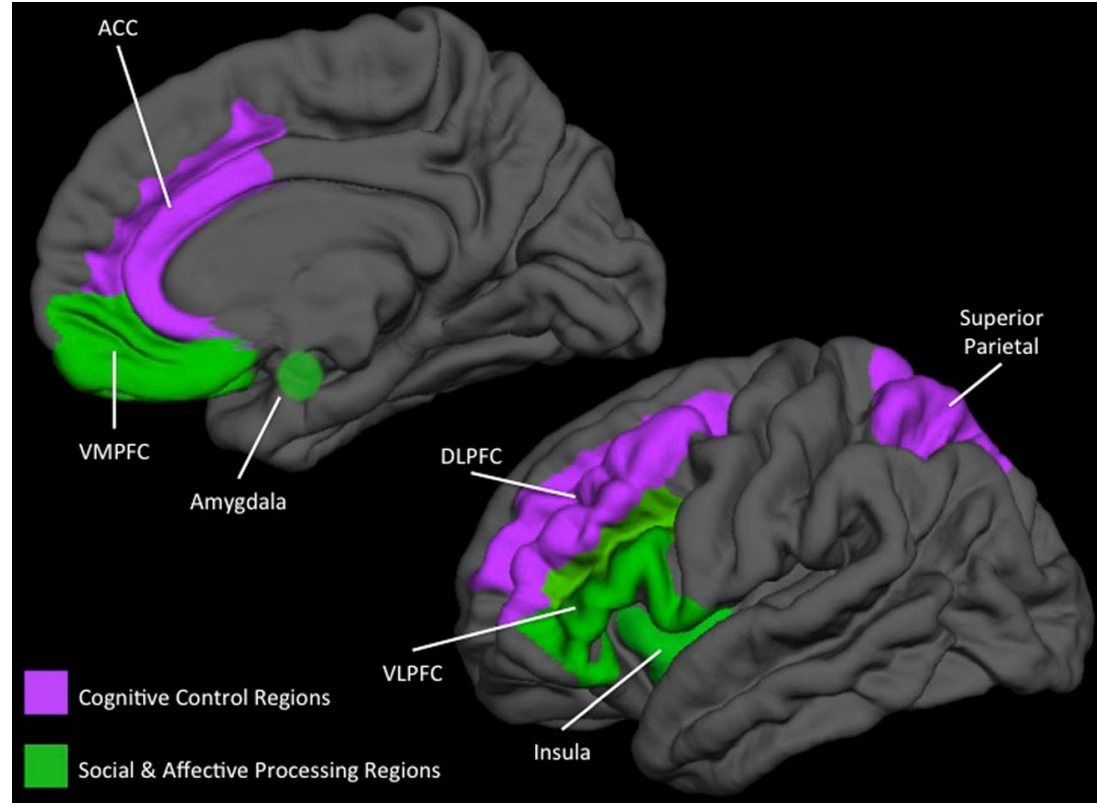

Questionnaire (CERQ) [75], are increasingly recognized as vulnerability factors that contribute to the development and maintenance of psychopathology [28], particularly disorders characterized by affective instability, such as anxiety [76], depression [77, 78], and substance use [79]. Similarly, impaired performance on ECA paradigms requiring inhibition of irrelevant negative emotional stimuli has been observed in depression [80], anxiety [81], borderline personality disorder [82], and those at risk for developing a mood disorder [83]. Moreover, dysfunctional activation in the frontal-limbic cognitive control network during ER and ECA has been linked to anxiety [84], depression [85], and risk for future depressive episodes [86]. Further, preliminary evidence also suggests a link to socially relevant behaviors, such as responding to interpersonal conflict: dysfunctional lateral prefrontal activation during affectively challenging tasks (e.g., viewing negative facial expressions of loved ones) relates to greater engagement in maladaptive behaviors in response to interpersonal conflict, such as increased rumination and substance use [56] - responses that could impact interpersonal relationships. However, this putative link between LPFC-mediated cognitive control of emotion mechanisms and social behavior has yet to be demonstrated directly.

Collectively, these data indicate that impairments in the ability to regulate responses to emotional and social stressors contribute to symptoms (particularly affective) across multiple disorders and could potentially impact socially relevant behaviors. Given that mood disturbance is pervasive across the psychosis spectrum, and that social functioning impairments are uniquely predictive of psychosis onset [13], researchers have begun to examine the role of cognitive control of emotion deficits in psychosis.

\section{Impaired Cognitive Control of Emotion in Psychosis-Spectrum Samples}

To date, investigations of cognitive control of emotion in psychosis-spectrum samples are limited and have predominantly been conducted in individuals high in psychosis proneness (PP) and individuals with a psychotic disorder, with a few studies in GHR samples and only one study in CHR. However, preliminary findings indicate that dysfunctions similar to those observed in mood and anxiety disorders are present across the psychosis spectrum.

Behavioral studies examining ER in PP samples indicate that reliance on maladaptive ER strategies, such as suppression, is associated with more severe psychotic symptoms, including paranoia [87] and auditory hallucinations [88], and that this effect may be more pronounced in the context of interpersonal stress [89]. Impaired emotional awareness and use of suppression is associated with worse social functioning in individuals with a diagnosis of schizophrenia [90-92] and CHR individuals [93•]. Moreover, these impairments appear to contribute to symptoms and social functioning impairments over and above neurocognitive deficits [91, 93•, 94]. Studies using ECA paradigms present similar findings. PP individuals demonstrate impaired inhibition of irrelevant negative faces during a flanker task [63], and increased negative word interference on the emotional Stroop task [95]. Similarly, GHR individuals show increased emotion interference effects on working memory performance, particularly in male subjects [96], and on emotional Stroop tasks [97]. In schizophrenia, impaired emotional Stroop performance relates to persecutory delusions [98], paranoia [99], and symptoms of disorganization [100]. Additionally, negative affective primes have been shown to negatively bias valence judgments of neutral 
Chinese characters [101], as well as social judgments of trustworthiness [102] - a direct example of how impaired cognitive control of emotion might relate to social processes.

Neuroimaging investigations parallel these findings and, consistent with the proposal that cognitive control of emotion deficits arise from dysfunctional activation in frontal-limbic circuitry, demonstrate reduced frontal-limbic connectivity during reappraisal in schizophrenia and bipolar patients [103] and in high-PP individuals [104]. Similarly, abnormal activation in the LPFC regions has been observed in schizophrenia patients during an emotional multisource interference task [105] and in PP individuals during the emotional Stroop task [106]. Moreover, Modinos and colleagues [107••] were able to distinguish between high- and low-PP individuals on the basis of neural activation in the frontal-limbic cognitive control of emotion network when viewing negative pictures. In PP individuals, failure to recruit lateral prefrontal regions when viewing socially accepting and rejecting faces has been shown to predict exacerbations of psychotic symptoms in response to interpersonal conflict [108••], further supporting the proposal that dysfunctional lateral prefrontal activation and reduced frontal-limbic connectivity is a psychosis vulnerability marker that impacts symptoms and adaptive responses to social stressors.

In summary, although studies examining cognitive control of emotion deficits in psychosis spectrum samples are limited, the findings indicate that impairments on ER and ECA paradigms, most likely caused by dysfunctional frontal-limbic connectivity, may be a key characteristic of the psychosis continuum that contributes to symptom onset and functional impairments. However, the contribution of cognitive control of emotion deficits to transition to a psychotic illness has yet to be examined. In order to directly tie this putative vulnerability marker to psychosis risk and outcomes, longitudinal prospective studies, especially in CHR samples, are necessary.

\section{Developmental Considerations}

The CHR state is most commonly observed in adolescents and young adults. Moreover, the prevalence of psychotic-like experiences in the general population is highest in adolescent individuals, and some of these experiences, such as suspiciousness and ideas of reference that are triggered during stressful social interactions, may be part of normal social development [109]. Given that adolescence is a period of extensive neurodevelopment, particularly of prefrontal structures and prefrontal-limbic connectivity [110, 111], and a period in which individuals master adaptive ER strategies such as positive reappraisal [112], it is possible that abnormal neurodevelopment of impaired cognitive control of emotion and the accompanying failure to develop adaptive ER strategies is part of the developmental pathophysiology of psychosis. Similarly, it is possible that successful development of cognitive control of emotion mechanisms and accompanying adaptive ER strategies during adolescence is one factor that distinguishes individuals who remit from the high-risk state from those who continue to meet CHR criteria. This is consistent with the neurodevelopmental theory of psychosis [113]; however, prospective longitudinal studies examining cognitive control of emotion in the context of neural and social development over the course of adolescence in high-risk samples are needed in order to answer this question.

\section{Treatment Implications}

Training individuals who tend to engage in maladaptive ER strategies to use more adaptive strategies could reduce symptoms and improve functioning. Positive reappraisal and problem solving, both considered adaptive ER strategies, are known to relate to lower depression and anxiety scores [76, 77], and there is growing evidence that cognitive therapy (CT), which emphasizes problem-solving and reappraisal skills, significantly reduces both positive and negative symptoms in CHR and first-episode patients [114-117]. However, whether CT can prevent transition to psychotic illness is still an open question [118]. Preliminary evidence indicates that training in adaptive ER strategies effects change at the neural level in frontal-limbic regions [119] and that normalization of LPFC-limbic activation, as a consequence of either medication [120] or CT [121], relates to decreased symptoms in depression and psychosis, respectively. Finally, a growing body of research suggests that computerized cognitive and social cognitive training can effect changes at both the neural and behavioral level in schizophrenia [122] and high-risk populations (see Fisher et al. [118]). Although these training paradigms are not specific to cognitive control of emotion and are still in the preliminary phases of clinical trials, they represent a new and exciting area of clinical intervention.

\section{Conclusions and Future Directions}

Cognitive control of emotion, encompassing the ability to regulate one's own emotional states and adjust the influence of emotional information on one's behavior, is essential for effective navigation of an inherently emotional and social world. Here we have reviewed converging lines of evidence and propose that impaired cognitive control of emotion, mediated by dysfunctional frontal-limbic networks, is a potential transdiagnostic mechanism underlying the heterogeneity of clinical presentation and psychosocial impairments observed in populations at high risk for psychosis. In brief, the current literature suggests the following: (1) emotion dysregulation is 
a core characteristic of psychosis-spectrum populations, as evidenced by the high prevalence of comorbid mood disorders in high-risk and psychotic samples; (2) impaired cognitive control of emotion is observable across the psychosis spectrum: maladaptive ER strategies are consistently observed in high-risk and psychotic samples, and ECA paradigms reveal an impaired ability to control the influence of irrelevant emotional information in schizophrenia, GHR, and PP samples; (3) emerging evidence suggests that cognitive control of emotion deficits relate to important clinical and psychosocial outcomes relevant to the high-risk state: maladaptive ER strategies contribute to symptoms and social functioning impairments in PP samples, and impairments on ECA paradigms - and the associated dysfunctional activation in frontal-limbic regions - relate to daily fluctuations in symptoms and socially relevant behavior in PP and psychotic samples; (4) preliminary investigations indicate treatment interventions aimed at improving cognitive control of emotion (e.g., CT) could reduce psychotic symptoms and improve functioning, possibly by effecting change at the neural level in frontal-limbic regions.

Collectively, the evidence reviewed here indicates impaired cognitive control of emotion may be a psychosis vulnerability marker that contributes to both psychotic and mood symptoms, and could be relevant to social functioning impairments observed early in the development of psychotic illness. However, the literature to date is fairly sparse, particularly in CHR samples; there remain many open questions regarding the role of cognitive control of emotion impairments in the transition to psychosis and long-term outcomes. Consequently, we highlight five key avenues for future research examining cognitive control of emotion as a psychosis vulnerability marker:

1. Studies examining cognitive control of emotion in CHR samples are needed. As evidenced by our summary of the literature to date, research examining cognitive control of emotion as a psychosis vulnerability marker has predominantly been conducted in PP samples, as well as a handful of studies in GHR individuals and those who already have a diagnosed psychotic disorder. To our knowledge, there is only one published study in CHR individuals [93•], which examined cognitive control of emotion indirectly via measures of emotional awareness, rather than directly testing ER or ECA. For cognitive control of emotion deficit to be identified as a vulnerability marker, it must be observable across the psychosis spectrum and in all high-risk populations [123], thus further investigations are needed before firm conclusions regarding its status as a vulnerability marker can be made. To maximize research progress, it will be important for the field to use a set of agreed-upon tasks for assessing cognitive control of emotion processes. This leads to our second recommendation for future research.
2. The development of specific tasks for testing frontallimbic function during cognitive control of emotion that can be used to detect impairments and are sensitive to change (i.e., can be used to assess treatment efficacy and illness trajectory) is necessary. This would help unify disparate lines of research and facilitate comparison across studies and neurodevelopmental stages. The Cognitive Neuroscience Treatment Research to Improve Cognition in Schizophrenia (CNTRICS) initiative has been successful in this mandate, developing cognitive neuroscience paradigms that tap neurocognitive biomarkers in psychosis that could serve as measures of treatment effects in translational settings [124]. In light of growing consensus that abnormalities in socioemotional processing are pervasive in psychosis, the CNTRICS initiative has begun to identify paradigms for the assessment of social and emotion-processing constructs [125]. This is still a relatively nascent area of research, however, with no paradigms ready for use in clinical trials. Continued development of valid and reliable tasks tapping socio-cognitive neural mechanisms, particularly in the context of cognitive control of emotion, will be essential for characterization of the psychosis risk state, identification of specific predictors of transition and long-term outcomes, and development of effective prevention and early intervention strategies.

3. Future research should examine impaired cognitive control of emotion in the context of positive ER. To date, investigations of cognitive control of emotion in psychosisspectrum samples have primarily focused on the inhibition of negative emotional information. However, given that cognitive control mechanisms are involved not just in inhibitory but also in facilitatory processes (e.g., generation and maintenance of goal representations [67]), impaired engagement of cognitive control mechanisms to downregulate negative affective information could be accompanied by a complementary deficit in the upregulation of positive affective information. Failure to upregulate positive emotion may underlie anticipatory pleasure deficits observed in psychosis [126-128] and CHR individuals [129], and could contribute to associated reward/motivational impairments thought to contribute to negative symptoms of anhedonia and avolition $[130,131]$. Conversely, failure to downregulate positive emotion may underlie approach-motivation and rewardprocessing abnormalities observed in affective psychoses such as bipolar disorder $[132,133]$. Future investigations could examine the differential contribution of inhibitory and facilitatory mechanisms of cognitive control of emotion to delineate this further.

4. Prospective longitudinal studies are necessary for understanding the interaction of frontal-limbic neurodevelopment, social development, and the emergence of cognitive control of emotion abilities, both in healthy samples and in the psychosis risk state. Emerging evidence in healthy samples demonstrates that LPFC activation during ER in childhood and adolescence 
is similar to activation observed in adults but increases linearly with age and is independent of emotional reactivity [134, 135]. This age-related increase in LPFC is coupled with decreases in limbic activation and overall increased frontallimbic connectivity [136], indicating that over the course of normal adolescent development, improved ER abilities are facilitated by frontal-limbic neurodevelopment. However, these data are all collected from cross-sectional healthy samples across varying age ranges; no studies have conducted similar examinations in high-risk samples, and no longitudinal research has been published. Longitudinal studies in high-risk populations are needed to identify potential abnormalities in the neurodevelopmental processes underlying the developmental of cognitive control of emotion abilities. Moreover, it will be important to examine relationships between abnormal development of neural mechanisms of cognitive control of emotion and psychosocial variables of interest, as this would tie the putative vulnerability to the clinical phenomenology of psychosis. This leads to our fifth, and final, suggestion for future research.

5. Finally, prospective studies that meaningfully connect neural indicators of mental processes (e.g., LPFC dysfunction during cognitive control of emotion) to experience sampling data collected regarding daily life functioning in high-risk samples will likely be key to furthering our understanding of the neural underpinnings of behavioral and clinical phenotypes in psychosis. A growing body of literature demonstrates the utility of this "brain-as-predictor" approach [137] for bridging the gap between neuroscience research and behavioral outcomes [138]. This may be particularly important for understanding social behavior, because many of the important aspects of social interactions (e.g., social conflict) cannot be realistically reproduced in laboratory settings, let alone in the solo environment of an MRI scanner. Experience sampling methods (ESM) are by no means new to psychosis research. Important contributions to our understanding of daily life functioning in schizophrenia have already been made in recent years, using the technique. For example, ESM revealed that visual hallucinations were reported more frequently than auditory hallucinations [139], delineated anticipatory and consummatory pleasure processes in patients with social anhedonia [140]), and illuminated the nuanced relationship between person-environment interactions and symptom fluctuations [141]. Research in healthy individuals clearly demonstrates the value of combining neuroimaging and ESM data for understanding neural mechanisms underlying social and self-regulatory behavior: activity in frontal-limbic regions has been shown to relate to momentary feelings of social rejection during daily social interactions [142], successful smoking cessation [137], and response to social conflict [56]. Extension of this methodology to research examining cognitive control of emotion mechanisms in the psychosis risk state would help directly tie the putative biomarker to the clinical phenomenology of the disease and could assist in the identification of processes specifically predictive of transition to psychotic illness.

\section{Compliance with Ethics Guidelines}

Conflict of Interest Tara Niendam received grants from the National Institutes of Health (NIH) and NARSAD [National Alliance for Research on Schizophrenia and Depression]/Brain Behavior Research Foundation. Laura Tully has no conflicts of interest.

Human and Animal Rights and Informed Consent This article does not contain any studies with human or animal subjects performed by the authors.

\section{References}

Papers of particular interest, published recently, have been highlighted as:

- Of importance

-• Of major importance

1. Large MM, Nielssen O. Violence in first-episode psychosis: a systematic review and meta-analysis. Schizophr Res. 2011;125(2-3):209-20.

2. Marshall $\mathrm{M}$ et al. Association between duration of untreated psychosis and outcome in cohorts of first-episode patients: a systematic review. Arch Gen Psychiatry. 2005;62(9):975-83.

3. Bora E et al. Cognitive deficits in youth with familial and clinical high risk to psychosis: a systematic review and meta-analysis. Acta Psychiatr Scand. 2014. doi:10.1111/acps.12261.

4. Shah JL, Tandon N, Keshavan MS. Psychosis prediction and clinical utility in familial high-risk studies: selective review, synthesis, and implications for early detection and intervention. Early Interv Psychiatr. 2013;7(4):345-60.

5. Gooding DC, Tallent KA, Matts CW. Clinical status of at-risk individuals 5 years later: further validation of the psychometric high-risk strategy. J Abnorm Psychol. 2005;114(1):170-5.

6. Lenzenweger MF. Schizotypy: an organizing framework for schizophrenia research. Curr Dir Psychol Sci (Wiley-Blackwell). 2006; 15(4):162-6.

7. Fusar-Poli $\mathrm{P}$ et al. The psychosis high-risk state: a comprehensive state-of-the-art review. JAMA Psychiatr. 2013;70(1):107-20.

8. Wood SJ et al. Progressive changes in the development toward schizophrenia: studies in subjects at increased symptomatic risk. Schizophr Bull. 2008;34(2):322-9.

9. Fusar-Poli P et al. Neuroanatomy of vulnerability to psychosis: a voxel-based meta-analysis. Neurosci Biobehav R. 2011;35(5): 1175-85.

10. Brewer WJ et al. Generalized and specific cognitive performance in clinical high-risk cohorts: a review highlighting potential vulnerability markers for psychosis. Schizophr Bull. 2006;32(3):53855.

11. Gur RC et al. Neurocognitive growth charting in psychosis spectrum youths. JAMA Psychiatry. 2014;71(4):366-74.

12. Meyer EC et al. The relationship of neurocognition and negative symptoms to social and role functioning over time in individuals at 
clinical high risk in the first phase of the North American Prodrome Longitudinal Study. Schizophr Bull. 2014.

13. Cannon TD et al. Prediction of psychosis in youth at high clinical risk: a multisite longitudinal study in North America. Arch Gen Psychiatry. 2008;65(1):28-37.

14. Yung AR et al. Risk factors for psychosis in an ultra high-risk group: psychopathology and clinical features. Schizophr Res. 2004;67(2):131-42.

15. Schlosser DA et al. Recovery from an at-risk state: clinical and functional outcomes of putatively prodromal youth who do not develop psychosis. Schizophr Bull. 2012;38(6):1225-33.

16. Fusar-Poli $\mathrm{P}$ et al. Comorbid depressive and anxiety disorders in 509 individuals with an at-risk mental state: impact on psychopathology and transition to psychosis. Schizophr Bull. 2014;40(1): 120-31.

17. Lee TY et al. Neurocognitive function as a possible marker for remission from clinical high risk for psychosis. Schizophr Res. 2014;153(1-3):48-53.

18. Addington $\mathrm{J}$ et al. At clinical high risk for psychosis: outcome for nonconverters. Am J Psychiatr. 2011;168(8):800-5.

19. Lin A, Nelson B, Yung AR. 'At-risk' for psychosis research: where are we heading? Epidemiol Psychiatry Sci. 2012;21(4): 329-34.

20. Meyer SE et al. The psychosis prodrome in adolescent patients viewed through the lens of DSM-IV. J Child Adolesc Psychopharmacol. 2005;15(3):434-51.

21.• Kelleher I, Cannon M. Whither the psychosis-neurosis borderline. Schizophr Bull. 2014;40(2):266-8. Reviews converging evidence suggesting there is not such a clear boundary between mood and psychotic disorders, and proposes a move toward an integrated dimensional system to better reflect overlapping symptoms in psychopathology.

22. Fusar-Poli $\mathrm{P}$ et al. At risk for schizophrenic or affective psychoses? A meta-analysis of DSM/ICD diagnostic outcomes in individuals at high clinical risk. Schizophr Bull. 2013;39(4):923-32.

23.• Krabbendam L, Hooker CI, Aleman A. Neural effects of the social environment. Schizophr Bull. 2014;40(2):248-51. Reviews epidemiological and neuroimaging research demonstrating the role of social stress in the shaping of neural circuits involved in emotion regulation and interpersonal functioning in the context of the diathesis-stress model of psychosis.

24.•van Os J, Murray RM. Can we identify and treat "schizophrenia light" to prevent true psychotic illness? BMJ. 2013;346:304. Argues that psychosis exists along a continuum and should be examined in the context of a clinical staging model emphasizing severity of symptoms across psychopathologies.

25. Cuthbert BN, Insel TR. Toward the future of psychiatric diagnosis: the seven pillars of RDoC. BMC Med. 2013;11(1):126.

26. Buckholtz JW, Meyer-Lindenberg A. Psychopathology and the human connectome: toward a transdiagnostic model of risk for mental illness. Neuron. 2012;74(6):990-1004.

27. Goschke T. Dysfunctions of decision-making and cognitive control as transdiagnostic mechanisms of mental disorders: advances, gaps, and needs in current research. Int J Methods Psychiatr Res. 2014;23 Suppl 1:41-57.

28. Gross JJ, John OP. Individual differences in two emotion regulation processes: implications for affect, relationships, and wellbeing. J Pers Soc Psychol. 2003;85(2):348.

29. Gross JJ, Muñoz RF. Emotion regulation and mental health. Clin Psychol Sci Pract. 1995;2(2):151-64.

30. Phillips LK, Seidman LJ. Emotion processing in persons at risk for schizophrenia. Schizophr Bull. 2008;34(5):888-903.

31. Ochsner KN, Gross JJ. The cognitive control of emotion. Trends Cogn Sci. 2005;9(5):242-9.
32. Gross JJ, Thompson RA. Emotion regulation: conceptual foundations. In: Gross JJ, editor. Handbook of emotion regulation. New York: Guilford; 2007. p. 3-24.

33. Koole SL. The psychology of emotion regulation: an integrative review. Cogn Emot. 2009;23(1):4-41.

34. Aleman A, Kahn RS. Strange feelings: do amygdala abnormalities dysregulate the emotional brain in schizophrenia? Prog Neurobiol. 2005;77(5):283-98.

35. Marwaha $\mathrm{S}$ et al. Mood instability and psychosis: analyses of British national survey data. Schizophr Bull. 2014;40(2): 269-77.

36. Verdoux $\mathrm{H}$ et al. Increased occurrence of depression in psychosisprone subjects: a follow-up study in primary care settings. Compr Psychiatry. 1999;40(6):462-8.

37. Achim AM et al. How prevalent are anxiety disorders in schizophrenia? A meta-analysis and critical review on a significant association. Schizophr Bull. 2011;37(4):811-21.

38. Majadas $\mathrm{S}$ et al. Prevalence of depression and its relationship with other clinical characteristics in a sample of patients with stable schizophrenia. Compr Psychiatry. 2012;53(2):145-51.

39. Masillo A et al. Interpersonal sensitivity in the at-risk mental state for psychosis. Psychol Med. 2012;42(09):1835-45.

40. Varghese D et al. Psychotic-like experiences in major depression and anxiety disorders: a population-based survey in young adults. Schizophr Bull. 2011;37(2):389-93.

41. Corcoran $\mathrm{CM}$ et al. The relationship of social function to depressive and negative symptoms in individuals at clinical high risk for psychosis. Psychol Med. 2011;41(2):251-61.

42. Freeman D, Garety PA. Connecting neurosis and psychosis: the direct influence of emotion on delusions and hallucinations. Behav Res Ther. 2003;41(8):923-47.

43. Birchwood M. Pathways to emotional dysfunction in first-episode psychosis. Br J Psychiatry. 2003;182(5):373-5.

44. Kelleher I et al. Clinicopathological significance of psychotic experiences in non-psychotic young people: evidence from four population-based studies. $\mathrm{Br} \mathrm{J}$ Psychiatry. 2012;201(1):26-32.

45. van Rossum I et al. Affective dysregulation and reality distortion: a 10-year prospective study of their association and clinical relevance. Schizophr Bull. 2011;37(3):561-71.

46. Wigman JTW et al. Evidence that psychotic symptoms are prevalent in disorders of anxiety and depression, impacting on illness onset, risk, and severity - implications for diagnosis and ultrahigh risk research. Schizophr Bull. 2012;38(2):247-57.

47. Arriaga XB, Rusbult CE. Standing in my partner's shoes: partner perspective taking and reactions to accommodative dilemmas. Personal Soc Psychol Bull. 1998;24(9):927-48.

48. Lopes PN et al. Emotion regulation and the quality of social interaction: does the ability to evaluate emotional situations and identify effective responses matter? J Pers. 2011;79(2):429-67.

49. Heatherton TF, Wagner DD. Cognitive neuroscience of selfregulation failure. Trends Cogn Sci. 2011;15(3):132-9.

50. Ochsner KN, Silvers JA, Buhle JT. Functional imaging studies of emotion regulation: a synthetic review and evolving model of the cognitive control of emotion. Ann N Y Acad Sci. 2012;1251(1):E1-24.

51. Lesh TA et al. Cognitive control deficits in schizophrenia: mechanisms and meaning. Neuropsychopharmacology. 2011;36(1): 316-38. Comprehensive review of converging lines of evidence regarding cognitive and neurobiological deficits in schizophrenia. It proposes that dysfunction in domain-general, superordinate cognitive control mechanisms contributes to the pathophysiology of higher cognitive deficits in schizophrenia.

52. Becker TM et al. Prefrontal dysfunction in first-degree relatives of schizophrenia patients during a Stroop task. Neuropsychopharmacology. 2008;33(11):2619-25. 
53. Fusar-Poli $\mathrm{P}$ et al. Abnormal frontostriatal interactions in people with prodromal signs of psychosis: a multimodal imaging study. Arch Gen Psychiatry. 2010;67(7):683-91.

54. Niendam TA et al. Impaired context processing as a potential marker of psychosis risk state. Psychiatry Res Neuroimaging. 2014;221(1):13-20.

55. Walker EF, Diforio D. Schizophrenia: a neural diathesis-stress model. Psychol Rev. 1997;104(4):667.

56. Hooker CI et al. Neural activity to a partner's facial expression predicts self-regulation after conflict. Biol Psychiatry. 2010;67(5): 406-13.

57. Kring AM, Werner KH. Emotion regulation and psychopathology. In: Feldman PPRS, editor. The regulation of emotion. Mahwah: Lawrence Erlbaum Associates; 2004. p. 359-85.

58. Quirk GJ, Beer JS. Prefrontal involvement in the regulation of emotion: convergence of rat and human studies. Curr Opin Neurobiol. 2006;16(6):723-7.

59. Gyurak A, Gross JJ, Etkin A. Explicit and implicit emotion regulation: a dual-process framework. Cogn Emot. 2011;25(3): 400-12. Presents a dual process model of emotion regulation, which integrates explicit (e.g., reappraisal) and implicit (e.g., inhibitory control) forms of emotion regulation.

60. Etkin A et al. Resolving emotional conflict: a role for the rostral anterior cingulate cortex in modulating activity in the amygdala. Neuron. 2006;51(6):871-82.

61. Bishop $\mathrm{S}$ et al. Prefrontal cortical function and anxiety: controlling attention to threat-related stimuli. Nat Neurosci. 2004;7(2):184-8.

62. Schulz KP et al. Does the emotional go/no-go task really measure behavioral inhibition? Convergence with measures on a nonemotional analog. Arch Clin Neuropsychol. 2007;22(2):151-60.

63. Tully LM, Lincoln SH, Hooker CI. Impaired executive control of emotional information in social anhedonia. Psychiatry Res. 2012;197(1-2):29-35.

64. Niendam TA et al. Meta-analytic evidence for a superordinate cognitive control network subserving diverse executive functions. Cogn Affect Behav Neurosci. 2012;12(2):241-68.

65. Kohn $\mathrm{N}$ et al. Neural network of cognitive emotion regulation - an ALE meta-analysis and MACM analysis. Neuroimage. 2014;87: 345-55.

66. Miller EK, Cohen JD. An integrative theory of prefrontal cortex function. Annu Rev Neurosci. 2001;24(1):167.

67. Miller EK. The prefrontal cortex and cognitive control. Nat Rev Neurosci. 2000;1(1):59-65.

68. Botvinick $\mathrm{MM}$ et al. Conflict monitoring and cognitive control. Psychol Rev. 2001;108(3):624-52.

69. MacDonald AW et al. Dissociating the role of the dorsolateral prefrontal and anterior cingulate cortex in cognitive control. Science. 2000;288(5472):1835.

70. Bunge SA et al. Dissociable contributions of prefrontal and parietal cortices to response selection. Neuroimage. 2002;17(3):1562-71.

71. Williams K, Jarvis B. Cyberball: a program for use in research on interpersonal ostracism and acceptance. Behav Res Methods. 2006;38(1):174-80.

72. Eisenberger NI, Lieberman MD, Williams KD. Does rejection hurt? An fMRI study of social exclusion. Science. 2003;302(5643):290-2.

73. Eisenberger NI et al. Neural pathways link social support to attenuated neuroendocrine stress responses. Neuroimage. 2007;35(4):1601-12.

74. Lieberman MD et al. Putting feelings into words affect labeling disrupts amygdala activity in response to affective stimuli. Psychol Sci. 2007;18(5):421-8.

75. Garnefski N, Kraaij V. The cognitive emotion regulation questionnaire. Eur J Psychol Assess. 2007;23(3):141-9.
76. Zlomke KR, Hahn KS. Cognitive emotion regulation strategies: gender differences and associations to worry. Personal Individ Differ. 2010;48(4):408-13.

77. Garnefski N, Kraaij V. Relationships between cognitive emotion regulation strategies and depressive symptoms: a comparative study of five specific samples. Personal Individ Differ. 2006;40(8):1659-69.

78. Garnefski N et al. Cognitive emotion regulation strategies and depressive symptoms: differences between males and females. Personal Individ Differ. 2004;36(2):267-76.

79. Aldao A, Nolen-Hoeksema S, Schweizer S. Emotion-regulation strategies across psychopathology: a meta-analytic review. Clin Psychol Rev. 2010;30(2):217-37.

80. Joormann J. Cognitive inhibition and emotion regulation in depression. Curr Dir Psychol Sci. 2010;19(3):161-6.

81. Eysenck MW et al. Anxiety and cognitive performance: attentional control theory. Emotion. 2007;7(2):336-53.

82. Silbersweig MDD et al. Failure of frontolimbic inhibitory function in the context of negative emotion in borderline personality disorder. Am J Psychiatr. 2007;164(12):1832-41.

83. Masland SR et al. Cognitive processing biases in individuals high on perceived criticism. Clinical Psychological Science. In press.

84. Bishop SJ. Trait anxiety and impoverished prefrontal control of attention. Nat Neurosci. 2009;12(1):92-8.

85. Fales CL et al. Altered emotional interference processing in affective and cognitive-control brain circuitry in major depression. Stress Depression Circuitry. 2008;63(4):377-84.

86. Hooley JM et al. Activation in dorsolateral prefrontal cortex in response to maternal criticism and praise in recovered depressed and healthy control participants. Biol Psychiatry. 2005;57(7):80912.

87. Westermann $\mathrm{S}$ et al. Maladaptive cognitive emotion regulation prospectively predicts subclinical paranoia. Cogn Ther Res. 2013;37(4):881-5.

88. Badcock JC, Paulik G, Maybery MT. The role of emotion regulation in auditory hallucinations. Psychiatry Res. 2011;185(3):303-8.

89. Westermann S, Kesting ML, Lincoln TM. Being deluded after being excluded? How emotion regulation deficits in paranoiaprone individuals affect state paranoia during experimentally induced social stress. Behav Ther. 2012;43(2):329-40.

90. Henry JD et al. Emotion regulation in schizophrenia: affective, social, and clinical correlates of suppression and reappraisal. J Abnorm Psychol. 2008;117(2):473.

91. Kimhy D et al. Emotion awareness and regulation in individuals with schizophrenia: implications for social functioning. Psychiatry Res. 2012;200(2-3):193-201.

92. Kimhy D et al. Emotional granularity and social functioning in individuals with schizophrenia: an experience sampling study. J Psychiatr Res. 2014;53:141-8.

93. van Rijn $\mathrm{S}$ et al. Affective dysfunctions in adolescents at risk for psychosis: emotion awareness and social functioning. Psychiatry Res. 2011;187(1-2):100-5. Proposes that emotion awareness is a prerequisite for emotion regulation and may underlie emotion regulation and social deficits in CHR individuals. CHR individuals demonstrated emotion awareness deficits that were independent of intellectual functioning and were related to social inadequacy and psychosis proneness.

94. Kim HS et al. Social cognition and neurocognition as predictors of conversion to psychosis in individuals at ultra-high risk. Schizophr Res. 2011;130(1-3):170-5.

95. Mohanty A et al. Specificity of emotion-related effects on attentional processing in schizotypy. Schizophr Res. 2008;103(1):129 37.

96. Phillips LK et al. Emotion-cognition interaction in people at familial high risk for schizophrenia: the impact of sex differences. J Abnorm Psychol. 2011;120(4):993. 
97. Besnier $\mathrm{N}$ et al. Stroop and emotional Stroop interference in unaffected relatives of patients with schizophrenic and bipolar disorders: distinct markers of vulnerability? World J Biol Psychiatr. 2009;10(4 Pt 3):809-18.

98. Bentall RP, Kaney S. Content specific information processing and persecutory delusions: an investigation using the emotional Stroop test. Br J Med Psychol. 1989;62:355-64.

99. Besnier $\mathrm{N}$ et al. Differential responses to emotional interference in paranoid schizophrenia and bipolar mania. Psychopathology. 2010;44(1):1-11.

100. Phillips LK et al. Emotional Stroop performance predicts disorganization in schizophrenia. Schizophr Res. 2005;77(2):141-9.

101. Suslow T, Roestel C, Arolt V. Affective priming in schizophrenia with and without affective negative symptoms. Eur Arch Psychiatry Clin Neurosci. 2003;253(6):292-300.

102. Hooker CI et al. Can I trust you? Negative affective priming influences social judgments in schizophrenia. J Abnorm Psychol. 2011;120(1):98-107.

103. Morris R et al. Lack of cortico-limbic coupling in bipolar disorder and schizophrenia during emotion regulation. Trans Psychiatry. 2012;2(3):e90.

104. Modinos G, Ormel J, Aleman A. Altered activation and functional connectivity of neural systems supporting cognitive control of emotion in psychosis proneness. Schizophr Res. 2010;118(1-3): 88-97.

105. Tully LM, Lincoln SH, Hooker CI. Lateral prefrontal cortex dysfunction during cognitive control of emotion predicts daily social experience in schizophrenia. Under review.

106. Mohanty A et al. Neural mechanisms of affective interference in schizotypy. J Abnorm Psychol. 2005;114(1):16-27.

107.• Modinos G et al. Multivariate pattern classification reveals differential brain activation during emotional processing in individuals with psychosis proneness. Neuroimage. 2012;59(3):3033-41. Used pattern classification analysis to differentiate high and low psychosis-prone individuals on the basis of frontal-limbic activation while they viewed negative affective pictures. Indicates that dysfunctional frontal-limbic activation during emotion processing is a psychosis vulnerability marker.

108.• Hooker CI et al. Neural activity to positive expressions predicts daily experience of schizophrenia-spectrum symptoms in adults with high social anhedonia. J Abnorm Psychol. 2014;123(1):190. Used functional MRI and experience sampling methods to demonstrate that lateral prefrontal cortex deficits in response to socially rejecting faces predict increased daily experience of psychotic symptoms in a sample of psychosis-prone individuals.

109. Wigman JTW et al. The structure of the extended psychosis phenotype in early adolescence-a cross-sample replication. Schizophr Bull. 2011;37(4):850-60.

110. Ingalhalikar $\mathrm{M}$ et al. Sex differences in the structural connectome of the human brain. Proc Natl Acad Sci. 2014;111(2):823-8.

111. Nelson EE et al. The social re-orientation of adolescence: a neuroscience perspective on the process and its relation to psychopathology. Psychol Med. 2005;35(2):163-74.

112. Aldwin CM. Stress, coping, and development: an integrative perspective. New York: Guilford; 2007.

113. Holtzman CW et al. Stress and neurodevelopmental processes in the emergence of psychosis. Neuroscience. 2013;249:172-91.

114. Addington $\mathrm{J}$ et al. A randomized controlled trial of cognitive behavioral therapy for individuals at clinical high risk of psychosis. Schizophr Res. 2011;125(1):54-61.

115. Morrison AP, Barratt S. What are the components of CBT for psychosis? A Delphi Study. Schizophr Bull. 2010;36(1): $136-42$.
116. Morrison AP et al. Cognitive therapy for the prevention of psychosis in people at ultra-high risk: randomised controlled trial. Br J Psychiatry. 2004;185(4):291-7.

117. Phillips LJ et al. Medium term follow-up of a randomized controlled trial of interventions for young people at ultra high risk of psychosis. Schizophr Res. 2007;96(1-3):25-33.

118. Fisher $\mathrm{M}$ et al. Cognitive interventions targeting brain plasticity in the prodromal and early phases of schizophrenia. Annu Rev Clin Psychol. 2013;9(1):435-63.

119. Goldin PR, Gross JJ. Effects of mindfulness-based stress reduction (MBSR) on emotion regulation in social anxiety disorder. Emotion. 2010;10(1):83-91.

120. Heller AS et al. Increased prefrontal cortex activity during negative emotion regulation as a predictor of depression symptom severity trajectory over 6 months. JAMA Psychiatr. 2013;70(11): 1181-9.

121. Edwards BG, Barch DM, Braver TS. Improving prefrontal cortex function in schizophrenia through focused training of cognitive control. Front Hum Neurosci. 2010;4:32.

122. Hooker CI et al. The influence of combined cognitive plus socialcognitive training on amygdala response during face emotion recognition in schizophrenia. Psychiatry Res Neuroimaging. 2013;213(2):99-107.

123. Gottesman II, Gould TD. The endophenotype concept in psychiatry: etymology and strategic intentions. Am J Psychiatr. 2003;160(4):636-45.

124. Carter CS et al. CNTRICS final task selection: social cognitive and affective neuroscience-based measures. Schizophr Bull. 2009;35(1):153-62.

125. Taylor SF, MacDonald AW. Brain mapping biomarkers of socioemotional processing in schizophrenia. Schizophr Bull. 2012;38(1):73-80.

126. Cohen AS et al. The state-trait disjunction of anhedonia in schizophrenia: potential affective, cognitive and social-based mechanisms. Clin Psychol Rev. 2011;31(3):440-8.

127. Kring AM, Moran EK. Emotional response deficits in schizophrenia: insights from affective science. Schizophr Bull. 2008;34(5): 819-34.

128. Pizzagalli D. The "anhedonia paradox" in schizophrenia: insights from affective neuroscience. Biol Psychiatry. 2010;67:899-901.

129. Fulford D et al. Symptom dimensions and functional impairment in early psychosis: more to the story than just negative symptoms. Schizophr Res. 2013;147(1):125-31.

130. Gold JM et al. Reward processing in schizophrenia: a deficit in the representation of value. Schizophr Bull. 2008;34(5):835-47.

131. Horan WP et al. Affective traits in schizophrenia and schizotypy. Schizophr Bull. 2008;34(5):856-74.

132. Green MJ, Cahill CM, Malhi GS. The cognitive and neurophysiological basis of emotion dysregulation in bipolar disorder. J Affect Disord. 2007;103(1-3):29-42.

133. Gruber J. A review and synthesis of positive emotion and reward disturbance in bipolar disorder. Clin Psychol Psychother. 2011;18(5):356-65.

134. Lévesque $\mathrm{J}$ et al. Neural basis of emotional self-regulation in childhood. Neuroscience. 2004;129(2):361-9.

135. McRae K et al. The development of emotion regulation: an fMRI study of cognitive reappraisal in children, adolescents and young adults. Soc Cogn Affect Neurosci. 2012;7(1):11-22.

136. Vink $\mathrm{M}$ et al. Functional differences in emotion processing during adolescence and early adulthood. Neuroimage. 2014;91:70-6.

137. Berkman ET, Falk EB, Lieberman MD. In the trenches of realworld self-control: neural correlates of breaking the link between craving and smoking. Psychol Sci. 2011;22(4):498-506.

138. Berkman ET, Lieberman MD. What's outside the black box? The status of behavioral outcomes in neuroscience research. Psychol Inq. 2011;22(2):100-7. 
139. Delespaul P. deVries M, van Os J. Determinants of occurrence and recovery from hallucinations in daily life. Social Psychiatry and Psychiatric. Epidemiology. 2002;37(3):97.

140. Gard DE et al. Anhedonia in schizophrenia: distinctions between anticipatory and consummatory pleasure. Schizophr Res. 2007;93(1-3):253-60.
141. Oorschot $\mathrm{M}$ et al. Momentary assessment research in psychosis. Psychol Assess. 2009;21(4):498-505.

142. Eisenberger NI, Gable SL, Lieberman MD. Functional magnetic resonance imaging responses relate to differences in real-world social experience. Emotion. 2007;7(4):745-54. 